\title{
Numerical Study of Heat Transfer by Natural Convection of Porous Layer Using Non-Equilibrium Model
}

\author{
Amir S. Dawood \\ Mohanad K. Radhi \\ Department of Mechanical Engineering, Mosul University
}

\begin{abstract}
This numerical study documents the phenomena of heat transfer by natural convection in thermally non-equilibrium porous cavity for Darcy flow model. The cavity is heated from below at constant temperature $\left(T_{h}\right)$ with keeping the upper plate isothermal $\left(T_{c}\right)$. The side walls were assumed to be thermally insulated. Finite difference method has been employed for discretization of governing equations which include a Darcian momentum equation and energy equations for solid and fluid phases. An iterative Guass-Seidle method was applied for determination of final solution of governing equations. This investigation was performed under the effect of the following non-dimensional groups: modified Rayleigh number $\left(0 \leq R a^{*} \leq 900\right)$, scaled heat transfer coefficient $(0.1 \leq H \leq 100)$ and thermal conductivity ratio $\left(0.1 \leq K_{r} \leq 100\right)$. The non-equilibrium is found to be affected by scaled heat transfer coefficient more than being affected by the thermal conductivity ratio. So It had been noticed that all Nusselt numbers are affected by the thermal conductivity ratio, while the effect of scaled heat transfer coefficient was confined to a solid phase Nusselt number.
\end{abstract}

Keywords: Porous Layer, Heated from Below, Thermal Non-equilibrium Model

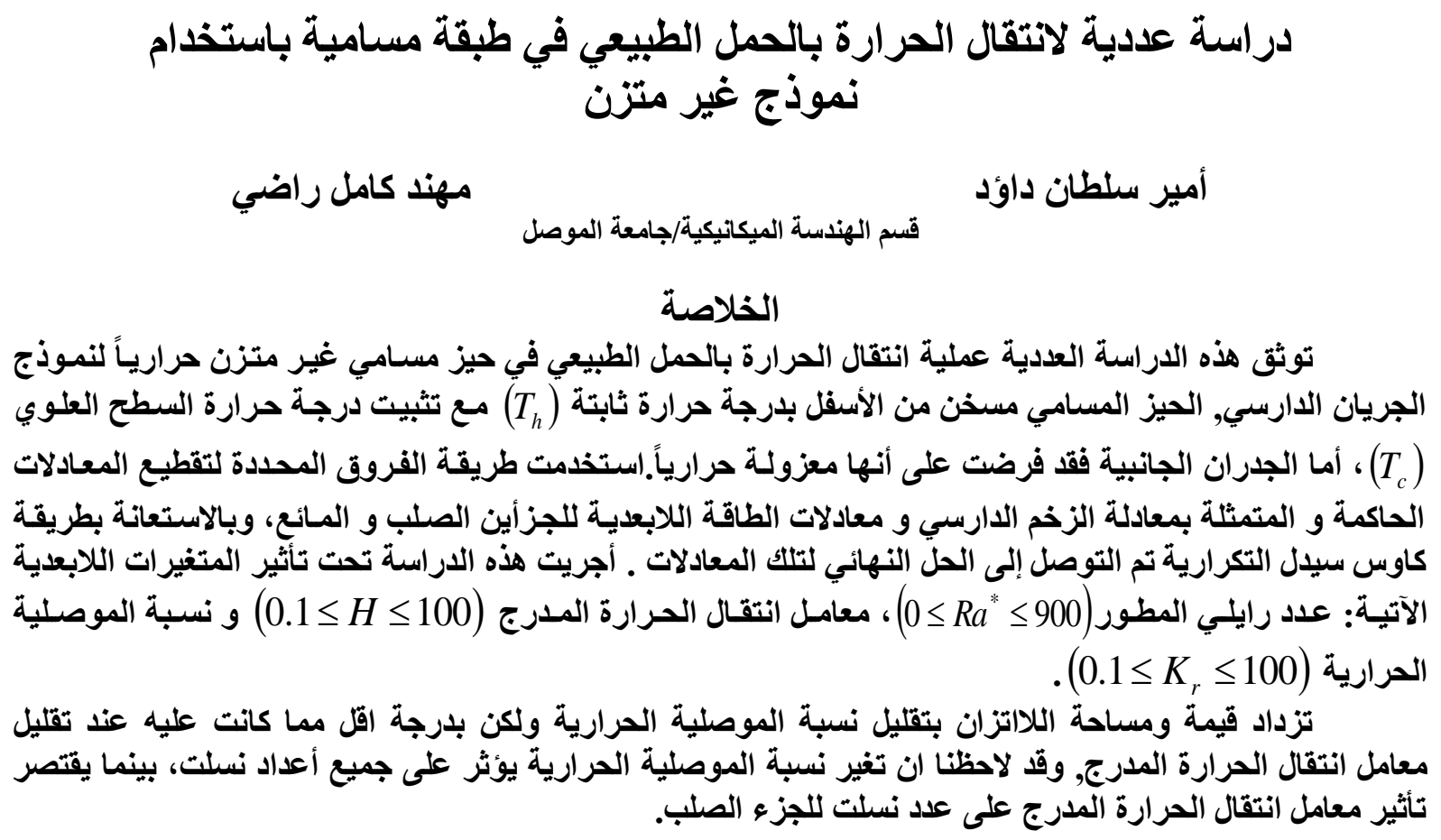

Received: $23-11-2010$

Accepted: 3 - 4 - 2011 
Nomenclature

\begin{tabular}{|c|c|}
\hline $\mathrm{C}$ & Specific thermal capacity,(J/kg.K) \\
\hline $\mathrm{D}$ & Height of porous cavity, (m) \\
\hline $\mathrm{Da}$ & Darcy number $=\left(K / H^{2}\right)$ \\
\hline G & Gravitational acceleration, $\left(\mathrm{m} / \mathrm{s}^{2}\right)$ \\
\hline $\mathrm{h}$ & Heat transfer coefficient, $\left(\mathrm{W} / \mathrm{m}^{2} \cdot \mathrm{K}\right)$ \\
\hline$h_{v}$ & Volumetric heat transfer coefficient, $\left(\mathrm{W} / \mathrm{m}^{3} . \mathrm{K}\right)$ \\
\hline $\mathrm{H}$ & Scaled heat transfer coefficient $=\left(h_{v} D^{2} / \phi \cdot k_{f}\right)$ \\
\hline $\mathrm{K}$ & Permeability of porous medium, $\left(\mathrm{m}^{2}\right)$ \\
\hline $\mathrm{k}$ & Thermal conductivity, $(W / m . K)$ \\
\hline $\mathrm{L}$ & Height of porous cavity, ( m) \\
\hline LTNE & Local thermal non-equilibrium $=\left(\left|T_{s}-T_{f} / T_{h}-T_{c}\right|\right)$ \\
\hline $\mathrm{Nu}$ & Nusselt number $=\left(q_{\text {total }} / q_{\text {conduction }}\right)$ \\
\hline $\mathrm{P}$ & Pressure, $\left(\mathrm{N} / \mathrm{m}^{2}\right)$ \\
\hline $\mathrm{Q}$ & Heat transfer, $(\mathrm{W})$ \\
\hline $\mathrm{T}$ & Temperature, $(\mathrm{K})$ \\
\hline $\mathrm{u}$ & Horizontal component of velocity, $(\mathrm{m} / \mathrm{s})$ \\
\hline $\mathrm{U}$ & Horizontal component of non-dimensional velocity \\
\hline $\mathrm{v}$ & Vertical component of velocity, $(\mathrm{m} / \mathrm{s})$ \\
\hline $\mathrm{V}$ & Vertical component of non-dimensional velocity \\
\hline $\mathrm{x}, \mathrm{y}$ & Cartesian coordinates $(\mathrm{m})$ \\
\hline $\mathrm{Y}, \mathrm{X}$ & Non-dimensional Cartesian coordinates \\
\hline$R a^{*}$ & Modified Rayleigh number $=\left(\rho_{f 0} g \beta K D \Delta T / \phi \mu \alpha_{f}\right)$ \\
\hline$K_{r}$ & Solid to fluid conductivity ratio \\
\hline
\end{tabular}

\section{Greek Symbols}

\begin{tabular}{|l|l|}
\hline$\mu$ & Dynamic viscosity $(\mathrm{kg} / \mathrm{m} . \mathrm{s})$ \\
\hline$\phi$ & Porosity \\
\hline$\alpha$ & Thermal diffusivity, $\left(\mathrm{m}^{2} / \mathrm{s}\right)$ \\
\hline$\rho$ & Density, $\left(\mathrm{kg} / \mathrm{m}^{3}\right)$ \\
\hline$\beta$ & Coefficient of thermal expansion, $(1 / \mathrm{K})$ \\
\hline$\psi$ & Stream function $\left(\mathrm{m}^{2} / \mathrm{s}\right)$ \\
\hline$\theta$ & Non-dimensional temperature $=\left(T-T_{c} / T_{h-} T_{c}\right)$ \\
\hline
\end{tabular}

\section{Subscripts and superscripts}

\begin{tabular}{|l|l|}
\hline S & Solid phase \\
\hline F & Fluid Phase \\
\hline H & Hot Surface \\
\hline C & Cold Surface \\
\hline$\prime$ & Local Value \\
\hline$\wedge$ & Non-dimensional \\
\hline
\end{tabular}




\section{Introduction:}

The local thermal equilibrium in porous media may be failed for the conditions that subjected to graduated changes between solid and fluid temperatures, which occur during the early stages of transportation processes. This also occur during the latest stages of transportation processes, such that occur at high speed flow and through a porous medium of high porosity where the time of thermal communication and the area of communication are insufficient to bring both phases to be in thermal equilibrium[1].

The heat generation in the fluid or in the solid phases is one of the most common applications of local thermal non-equilibrium, so the differences in thermal conductivities or the thermal capacities of both phases can lead to local thermal non-equilibrium.

The investigation of thermal parameters of two phase systems is valuable in many industrial fields, such as explosive industry, ceramic industry, nuclear reactors and rocket and projectile technology[2].

Many researchers studied the problem of local thermal non-equilibrium in porous medium, i.e. Minkowycs et al [3] studied the effect of Sparrows number on local thermal non-equilibrium in porous layer heated from below by sinusoidal heat flux, their results showed that the increase of Sparrows number over than 100 makes the system reaches to local thermal equilibrium rapidly. Haddad et al. [4] did analytical study to examine the validity of thermal equilibrium for the natural convection from vertical plate immersed in porous medium with non-Darcian flow. The results demonstrated that there are four parameters control the thermal equilibrium assumption: volumetric Biot number $\left(\mathrm{Bi}_{\mathrm{v}}\right)$, modified Rayleigh number ( $\mathrm{Ra}^{*}$ ), Darcy number(Da) and effective dynamic viscosity ratio $\left(\lambda_{\text {eff }}\right)$. The area of local thermal non-equilibrium is proportionally increase with the increase of all parameters except modified Rayleigh number. Nanna et al. [5] did a practical investigation to study the thermal non-equilibrium in porous medium saturated with a fluid subjected to phase change effect. Their model was insulated box made from insulated material called (Plexiglas) and filled with glass balls of $(3 \mathrm{~mm})$ diameter immersed in (Tetracosane). The results demonstrated the validity of local thermal non-equilibrium for the early stages of heat transfer, then, the local thermal equilibrium satisfied when Sparrow number over than 100.

The present work focus on the local thermal non-equilibrium in a porous cavity heated from below as shown in (fig.1), the lower and upper walls at temperatures $T_{h}$ and $T_{c}$ respectively, the other two walls are thermally insulated, and the flow is steady and according to Darcy model.

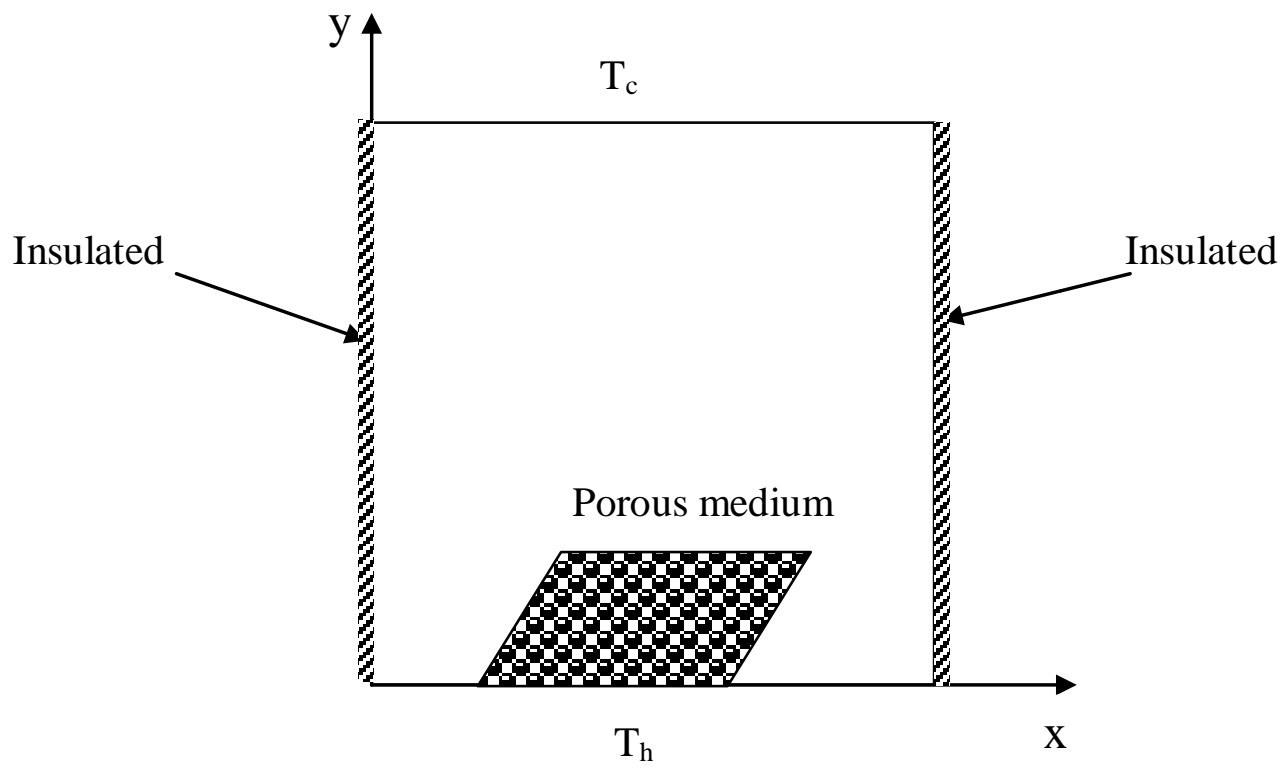

Fig(1):Schematic diagram of the problem. 


\section{Mathematical model and numerical solution}

The physical model of the present problem is subjected to many assumptions such as steady state heat transfer and fluid flow, Darcy model is applied to fluid flow. The fluid phase and solid phase temperatures are not same locally, and the thermal properties of solid phase are different from that for fluid phase. The thermal properties of fluid phase assumed to be constant except for the density change with temperature according to Boussensq approximation. All of cavity walls assumed to be impermeable.

The governing equations represented by: continuity, momentum, fluid phase energy transport and solid phase energy transport in tow dimensional steady state conditions as the following[6]:

$$
\begin{aligned}
& \frac{\partial u}{\partial x}+\frac{\partial v}{\partial y}=0 \\
& \frac{\partial u}{\partial y}-\frac{\partial v}{\partial x}=-\frac{\rho_{f_{o}} g \beta K}{\mu} \frac{\partial T_{f}}{\partial x} \\
& \left(\rho c_{p}\right)_{f}\left(u \frac{\partial T_{f}}{\partial x}+v \frac{\partial T_{f}}{\partial y}\right)=\phi k_{f}\left(\frac{\partial^{2} T_{f}}{\partial x^{2}}+\frac{\partial^{2} T_{f}}{\partial y^{2}}\right)+h_{v}\left(T_{s}-T_{f}\right) \\
& (1-\phi) k_{s}\left(\frac{\partial^{2} T_{s}}{\partial x^{2}}+\frac{\partial^{2} T_{s}}{\partial y^{2}}\right)+h_{v}\left(T_{f}-T_{s}\right)=0
\end{aligned}
$$
variables:

The governing equations are non-dimensionalized by defining the following groups of

$$
X=\frac{x}{D}, \quad Y=\frac{y}{D}, \quad A=\frac{L}{D}
$$

$\theta_{f}=\frac{T_{f}-T_{c}}{T_{h}-T_{c}}, \theta_{s}=\frac{T_{s}-T_{c}}{T_{h}-T_{c}}$

$$
\left\{\begin{array}{l}
u=\frac{D}{\alpha} * \frac{\partial \hat{\psi}}{\partial Y} \\
v=-\frac{D}{\alpha} * \frac{\partial \hat{\psi}}{\partial X}
\end{array}\right\} \ldots \ldots \ldots \ldots \ldots
$$

The non-dimensional continuity, momentum, fluid phase and solid phase energy equations expressed respectively as the following:

$$
\begin{aligned}
& \frac{\partial^{2} \hat{\psi}}{\partial X \partial Y}-\frac{\partial^{2} \hat{\psi}}{\partial X \partial Y}=0 \ldots \ldots \ldots \\
& \frac{\partial^{2} \hat{\psi}}{\partial X^{2}}+\frac{\partial^{2} \hat{\psi}}{\partial Y^{2}}=-R a^{*} \frac{\partial \theta_{f}}{\partial X}
\end{aligned}
$$


$\frac{\partial \widehat{\psi}}{\partial Y} \cdot \frac{\partial \theta_{f}}{\partial X}-\frac{\partial \widehat{\psi}}{\partial X} \cdot \frac{\partial \theta_{f}}{\partial Y}=\left(\frac{\partial^{2} \theta_{f}}{\partial X^{2}}+\frac{\partial^{2} \theta_{f}}{\partial Y^{2}}\right)+H\left(\theta_{s}-\theta_{f}\right)$

$\frac{\partial^{2} \theta_{s}}{\partial X^{2}}+\frac{\partial^{2} \theta_{s}}{\partial Y^{2}}+H \cdot K_{r}\left(\theta_{f}-\theta_{s}\right)=0$

Where $R a^{*}=.(D a)(R a)=\left(\frac{K}{D^{2}}\right) \cdot\left(\frac{\rho_{f o} g \beta D^{3} \Delta T}{\phi \mu \alpha_{f}}\right)=\frac{\rho_{f o} g \beta K D \Delta T}{\phi \mu \alpha_{f}}$ is the modified Rayleigh number, $H=\frac{h_{v} D^{2}}{\phi k_{f}}$ is the scaled heat transfer coefficient and $K_{r}=\frac{\phi k_{f}}{(1-\phi) k_{s}}$ is the thermal conductivity ratio, $k_{s}$ and $k_{f}$ are solid and fluid thermal conductivities.

The boundary conditions of the present problem is considered as the following:

at : $Y=0, \quad 0 \leq X \leq A$

$\theta_{s}=\theta_{f}=1, \quad \hat{\psi}=V=0$

at $: Y=1,0 \leq X \leq A$

$\theta_{s}=\theta_{f}=0, \widehat{\psi}=V=0$

at : $X=0,0 \leq Y \leq 1$

$\frac{\partial \theta_{s}}{\partial X}=\frac{\partial \theta_{f}}{\partial X}=0, \hat{\psi}=U=0$

at $: X=A, \quad 0 \leq Y \leq 1$

$\frac{\partial \theta_{s}}{\partial X}=\frac{\partial \theta_{f}}{\partial X}=0, \quad \widehat{\psi}=U=0$

The average fluid, solid, and total Nusselt numbers are calculated respectively as the following:

$$
\begin{aligned}
& N u_{f}=-\left.\frac{1}{A} \int_{0}^{A} \frac{\partial \theta_{f}}{\partial Y}\right|_{Y=0} d x, \quad N u_{s}=-\left.\frac{1}{A} \int_{0}^{A} \frac{\partial \theta_{s}}{\partial Y}\right|_{Y=0} d x, \\
& N u_{T}=-\frac{1}{A\left(1+K_{r}\right)} \int_{0}^{A}\left\{K_{r}\left(\frac{\partial \theta_{f}}{\partial Y}\right)_{Y=0}+\left(\frac{\partial \theta_{s}}{\partial Y}\right)_{Y=0}\right\} d x
\end{aligned}
$$

The finite difference method was employed to solve the non-dimensional governing equations, second order finite differences approximation were used to obtain the numerical form of the partial derivatives in governing equations, the central difference approximation is used for the interior nodes, while the forward and backward differences approximation are used for the boundary nodes.

After arranging the governing equations, a computer code written in FORTRAN language was prepared specially for solving this problem, the (over and under relaxation) technique was employed to hasten the speed of convergence, the criterion of convergence was assumed to satisfy the following condition: 


$$
\sum_{i=1}^{I X-1} \sum_{j=1}^{J Y-1} \frac{\left|\theta_{f(i, j)}^{n+1}-\theta_{f(i, j)}^{n}\right|}{\theta_{f(i, j)}^{n+1}} \leq 10^{-5}
$$

Many grid sizes were tested to satisfy minimum error between the lower and upper Nusselt numbers, the best grid size was found to be $(42 * 42)$.

The resulting values of average Nusselt number were compared with Kuriakoz's work for the thermal equilibrium at porous cavity heated from below case and showed good agreement.

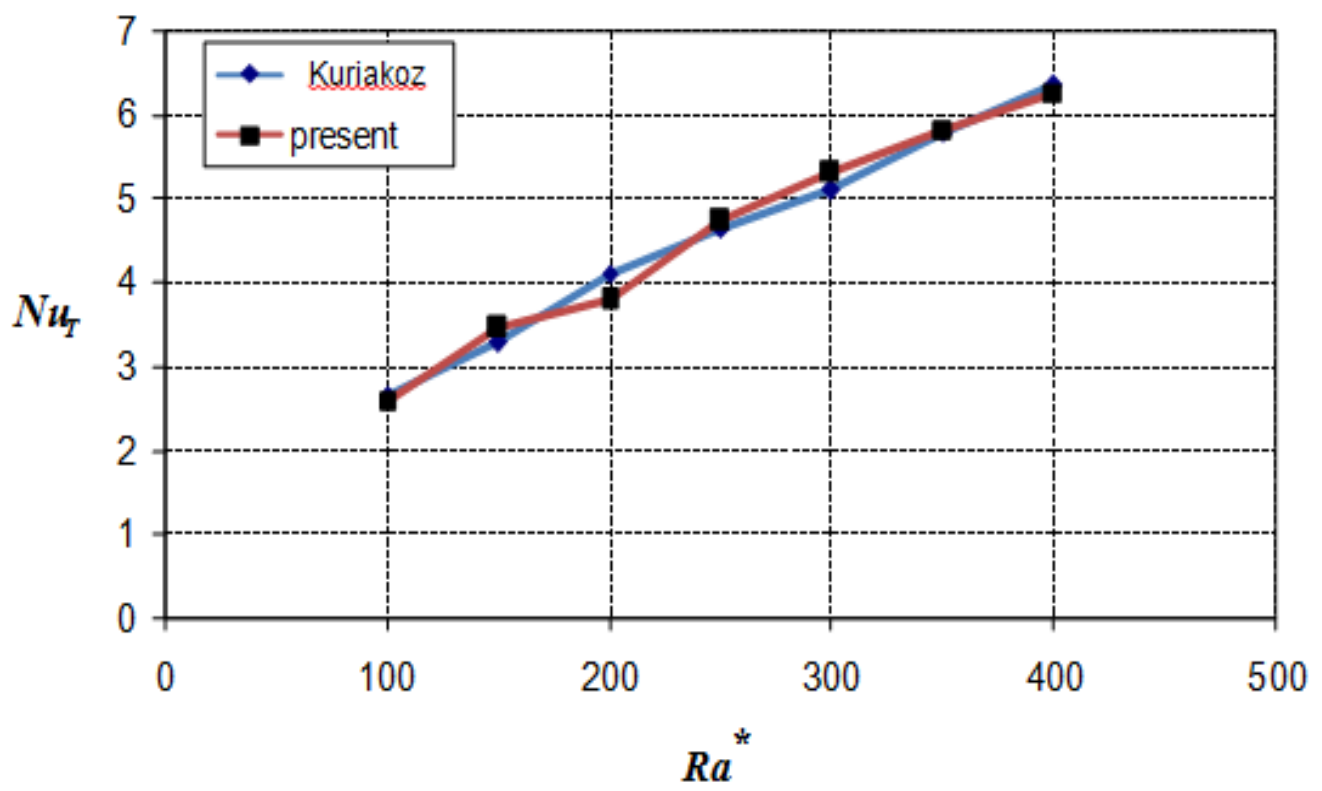

Fig.(2): The comparison of total Nusselt number of the present work at thermal equilibrium condition with Kuriakoz[7].

\section{Results and discussion}

The results of the current problem comprised the temperatures distribution, streamlines, the local thermal non equilibrium and Nusselt numbers. The results are strongly dependent of the problem parameters(i.e. Rayleigh number: $0<R a^{8}<900$, scaled heat transfer coefficient: $0.1<H<100$ and thermal conductivity ratio: $0<K_{r}<100$ ). The results presented graphically with the aid of curve fitting.

\section{Isotherms and streamlines}

Fig.(3) shows the effect of the scaled heat transfer coefficient on both streamlines and temperature distribution for both phases at $R a^{*}=100$. The reduction of scaled heat transfer causes a difference between solid phase temperature and fluid phase temperature, this occurs due to the reduction in heat exchange between the solid phase and the fluid phase due to the increase of convection resistance between the phases.

Fig.(4) demonstrate the effect of thermal conductivity ratio at $R a^{*}=100$, it is noticed that the reduction of thermal conductivity ratio leads to decrease the number of flow cells due to the weakness of buoyancy forces, the reduction in buoyancy forces continues until 
reaching the pure conduction state, and hence the local thermal equilibrium is applied to the system.
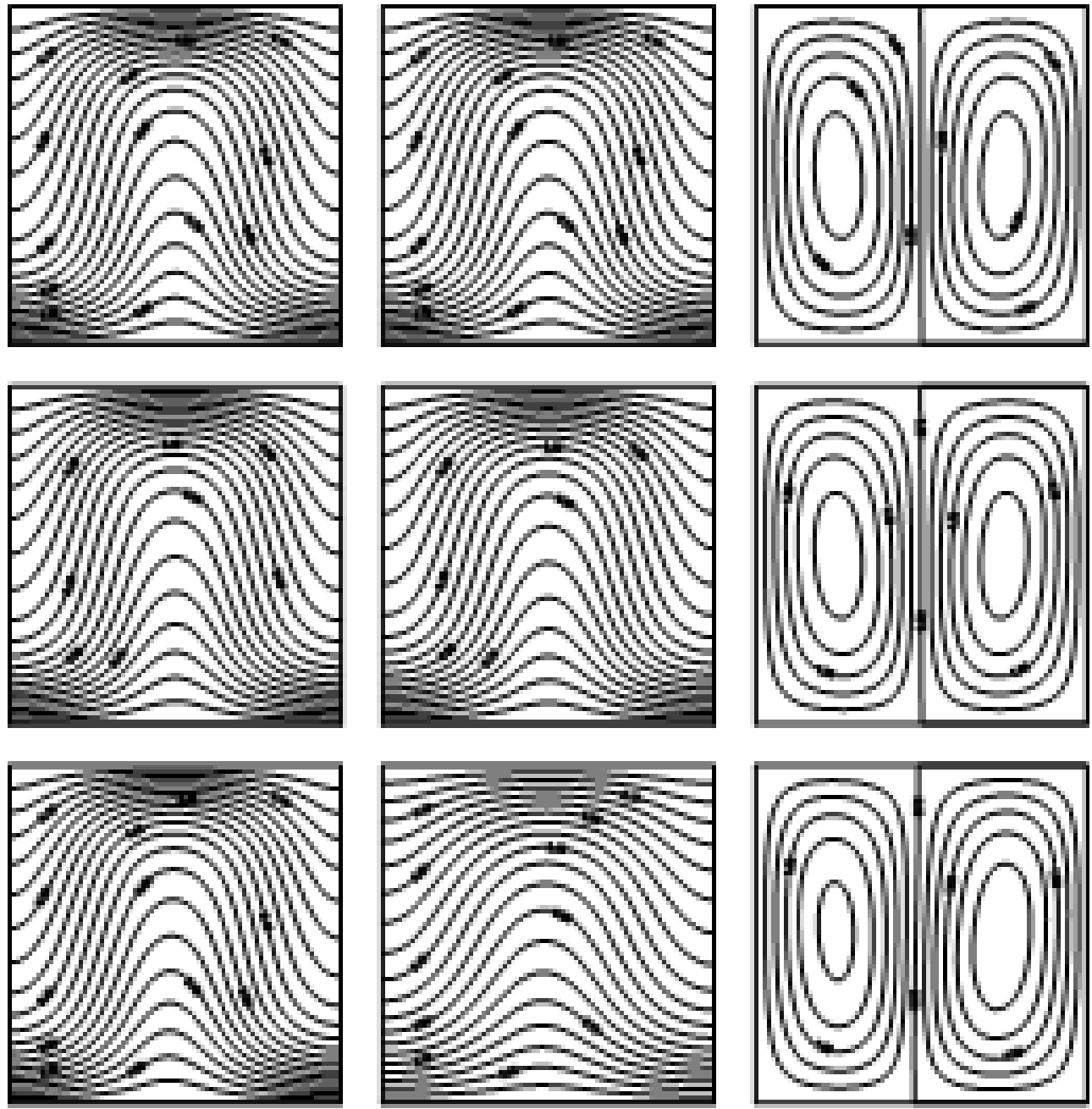

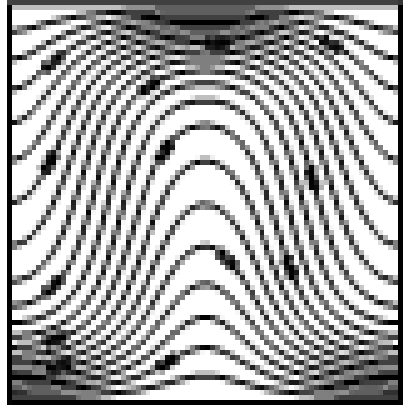

(a)

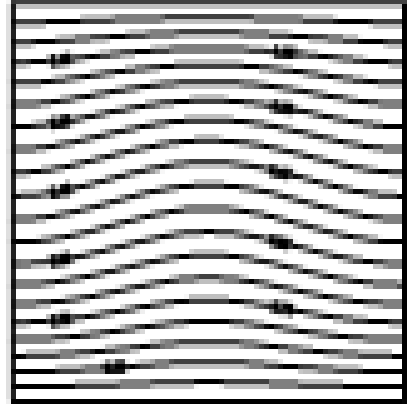

(b)

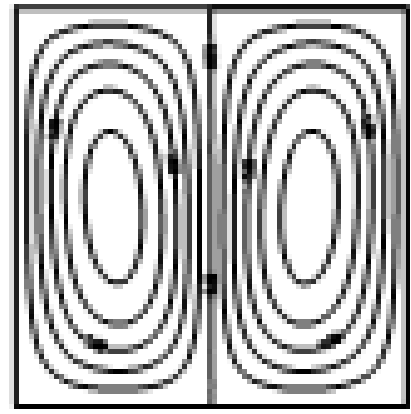

(c)

Fig.(3): Fluid phase isotherms (a), solid phase isotherms (b) and streamlines (c) at: $R a^{*}=100, K_{r}=100$ and $\mathrm{H}=100,10,1.0$ and 0.1 from up to down respectively 
(b)
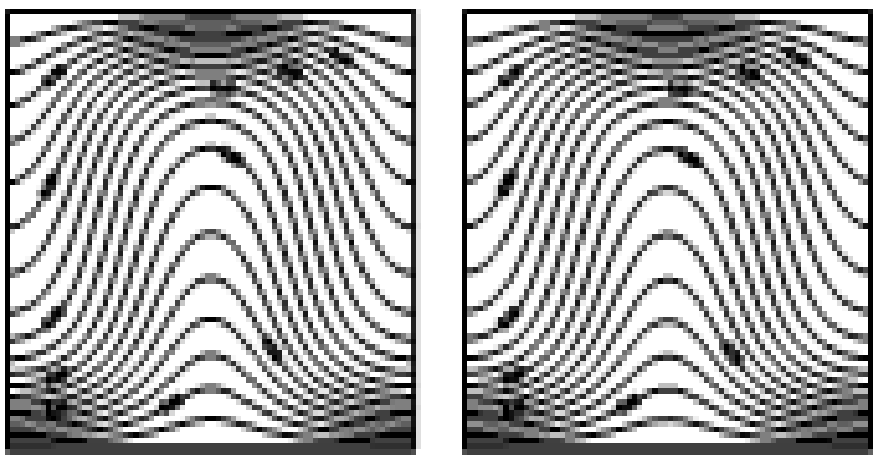

(b)

(c)
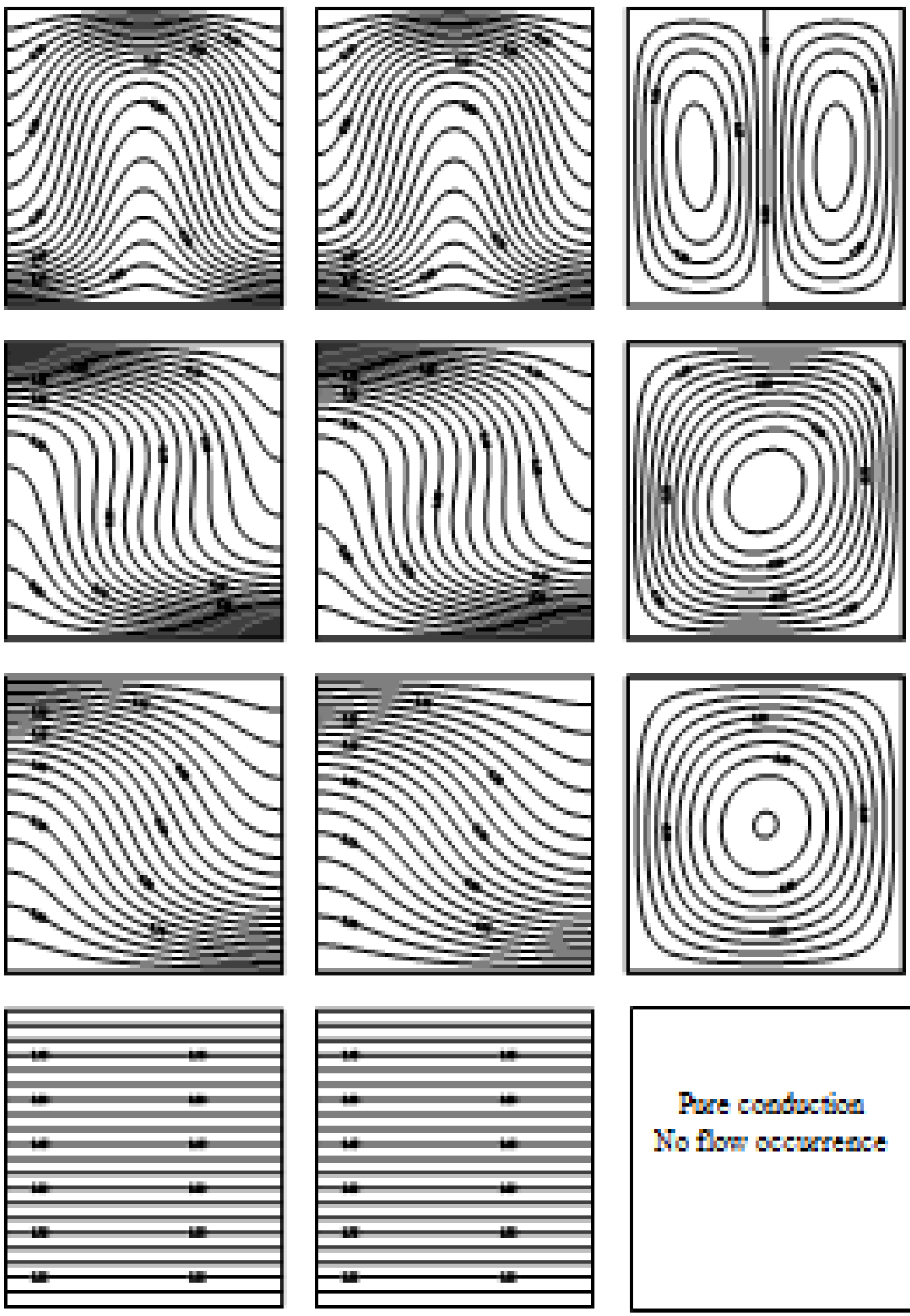

( a )

( b )

(c)

Fig.(4): Fluid phase isotherms (a), solid phase isotherms (b) and streamlines (c), at $R a^{*}=100, \mathrm{H}=100$ and $K_{r}=50,10,1.0,0.1$ from up to down respectively 
Fig.(5) shows a similar behavior of streamlines and isotherms under the effect of scaled heat transfer coefficient at $R a^{*}=900$, the gradient of the solid phase temperature is decreased due to the increase in scaled heat transfer coefficient
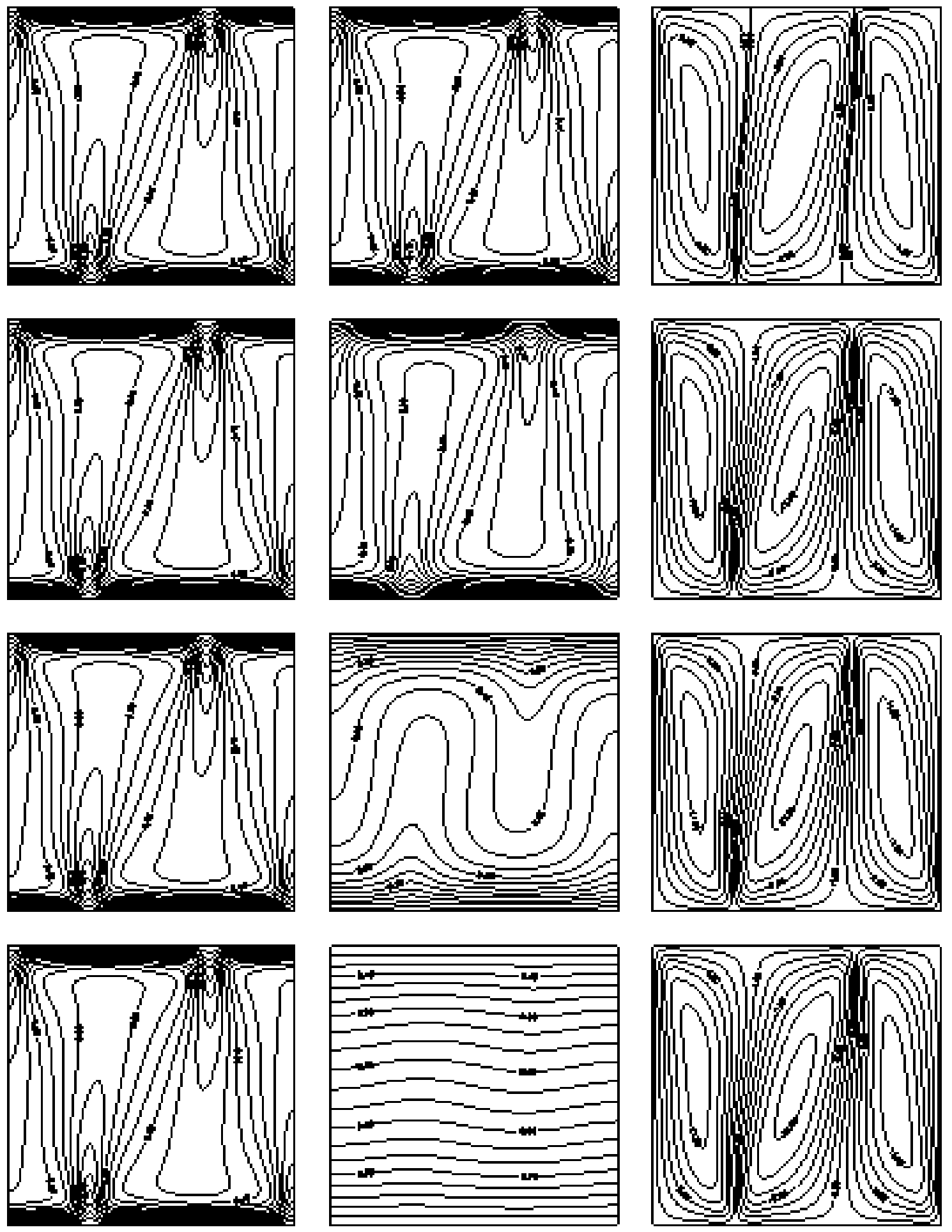

( a )

( b )

(c)

Fig.(5): Fluid phase isotherms (a), solid phase isotherms (b) and streamlines (c) at: $R a^{*}=900, K_{r}=100$ and $\mathrm{H}=100,10,1.0$ and 0.1 from up to down respectivelyin scaled heat transfer coefficient. 
We notice from fig(6) that the reduction in thermal conductivity ratio (at $R a^{*}=900$ ) leads to increase of the flow velocity because of that the decrease of thermal conductivity ratio can be made by reducing the porosity and hence the area of the flow will decrease.
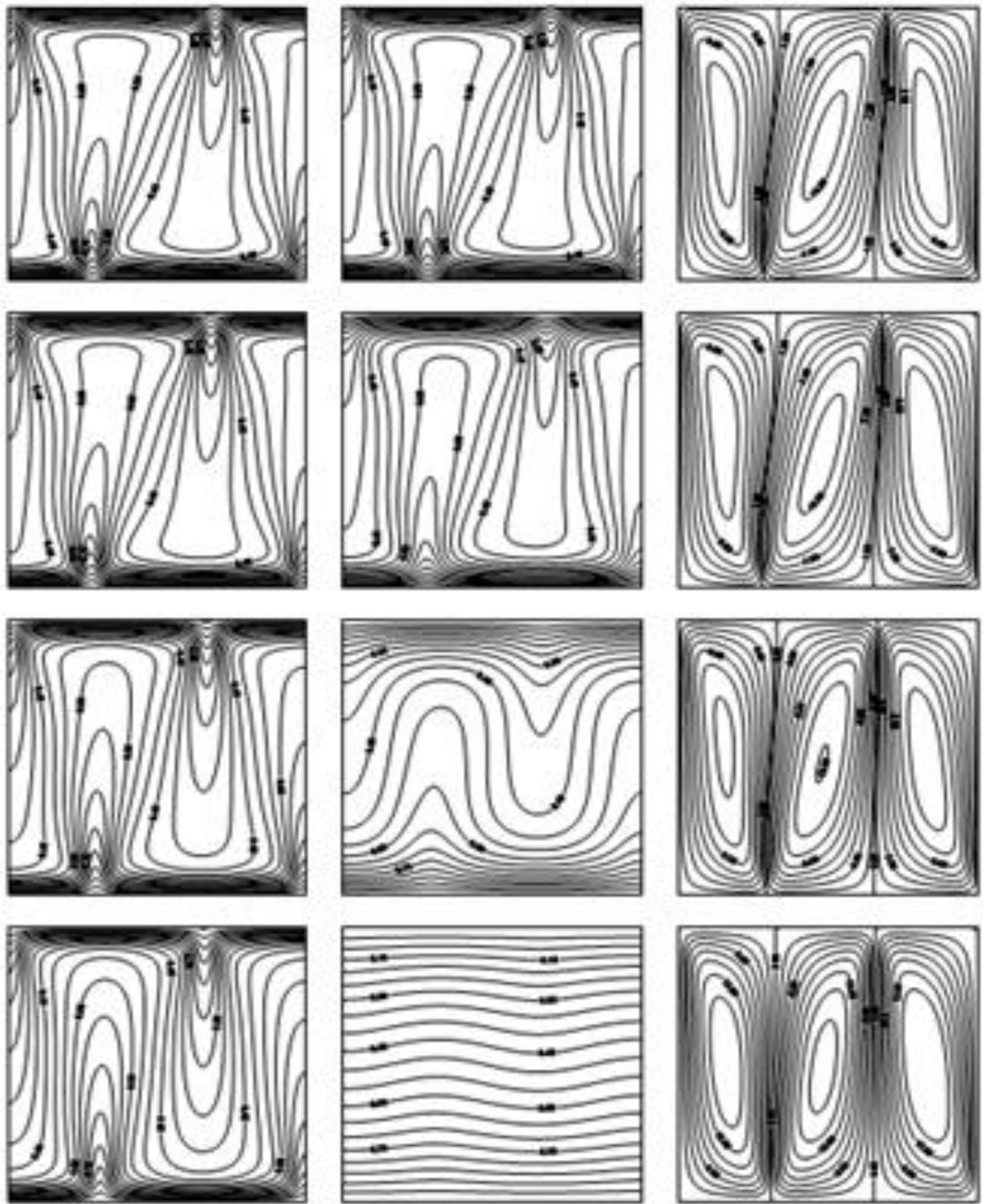

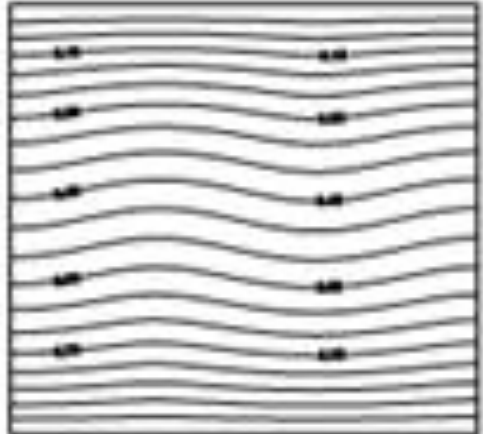

( b )

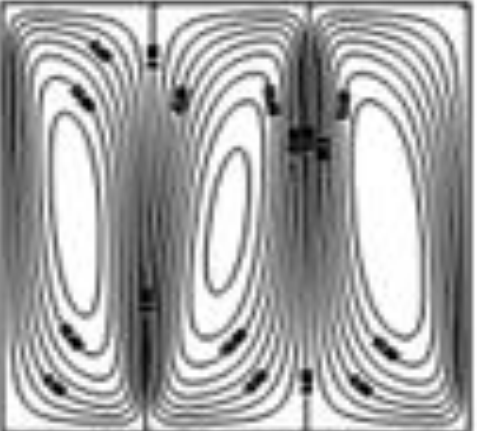

( c )

Fig.(6): Fluid phase isotherms (a), solid phase isotherms (b) and streamlines (c) at: $R a^{*}=900, \mathrm{H}=100$ and $K_{r}=50,10,1.0,0.1$ from up to down respectively 


\section{Local Thermal Non-Equilibrium}

The values of local thermal non-equilibrium (LTNE) were calculated by finding the absolute difference between the temperatures of solid and fluid phase locally. The critical value of local thermal non-equilibrium must not exceed one percent $(L T N E \leq 0.01)$ in order to make the region in thermal equilibrium, if LTNE exceeded the critical value, the region is considered to be thermally non-equilibrium. The critical value of LTNE is presented by black line, the black line represents the borders between the thermal equilibrium and the nonequilibrium. The blue color represents the minimum value of LTNE, while the rosy color represents the maximum value of LTNE as shown in figures (7and8).

Fig.(7) shows the effect of scaled heat transfer coefficient (column-a) and the effect of thermal conductivity ratio (columnb) at $R a^{*}=100$, the maximum values of LTNE occur where the ascending flow conflict with the upper plate, so the maximum values of LTNE occur where the descending flow conflict with the lower plate, this is due to the strong effect of conduction at that regions, this coincides with the stated at [7]. The hot fluid coming from the lower plate is conflict with the cold solid matrix nearby the upper plate causing the maximum value of the LTNE, in same manner, the cold fluid descending from upper plate is conflict with the lower plate causing maximum value of LTNE. The area and value of LTNE are proportionally increase with the decrease of both thermal conductivity ratio and scaled heat transfer coefficient except for $k_{r}=0.1$ where the cavity is completely under thermal equilibrium due to the pure conduction.
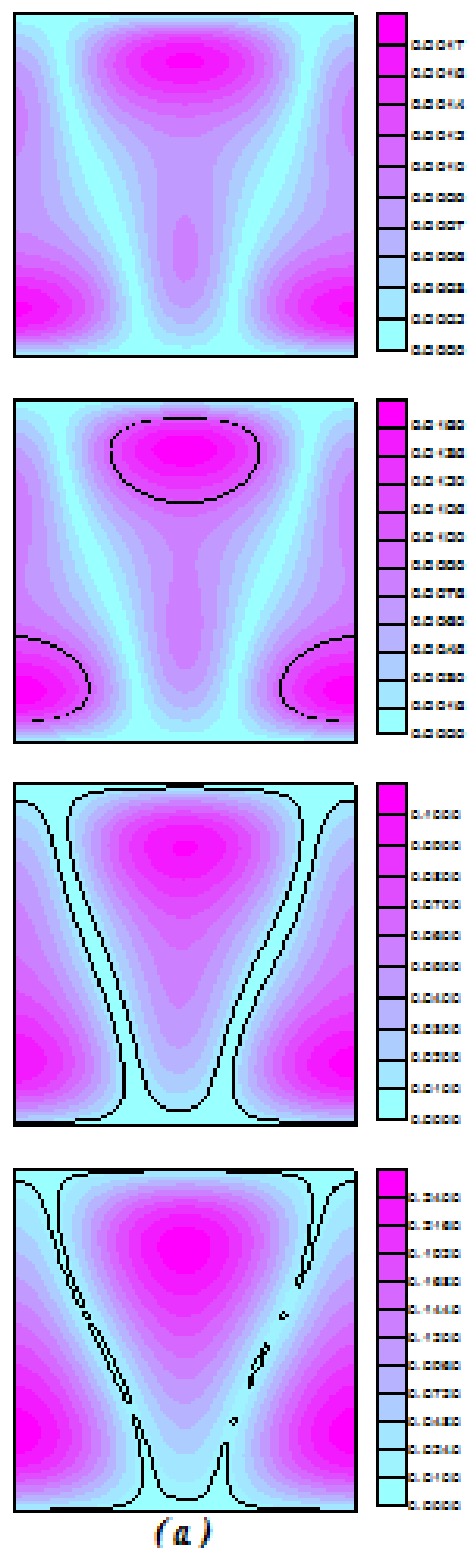
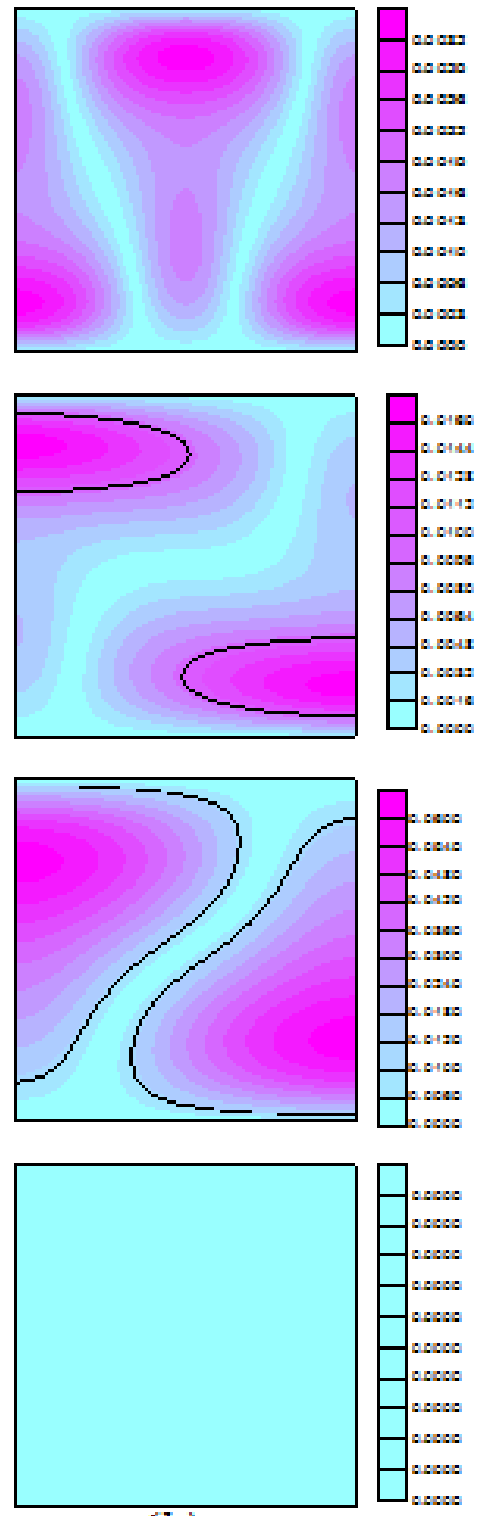

(b)

Fig.(7): The local thermal non-equilibrium (LTNE) at $R a^{*}=100$ for the conditions:

(a): $K_{r}=100$ and $\mathrm{H}=100,10,1.0$ and 0.1 from up to down respectively.

(b): $\mathrm{H}=100$ and $K_{r}=50,10,1.0$ and 0.1 from up to down respectively. 
Fig.(8) shows same behavior of LTNE to that happened at $R a^{*}=100$, but we noticed more increase in LTNE values due to the increase of buoyancy forces which leads to raise the flow velocity and hence the time of thermal communication between the solid particles and the fluid phase become shorter.
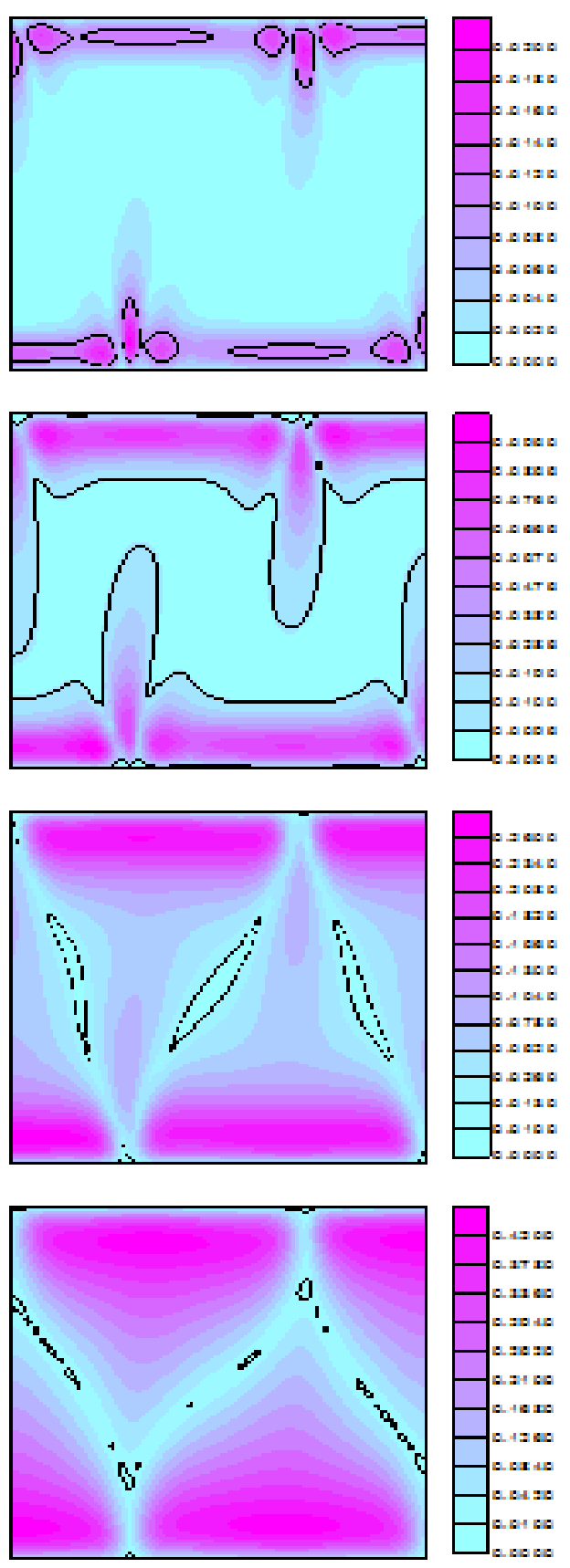

( a )
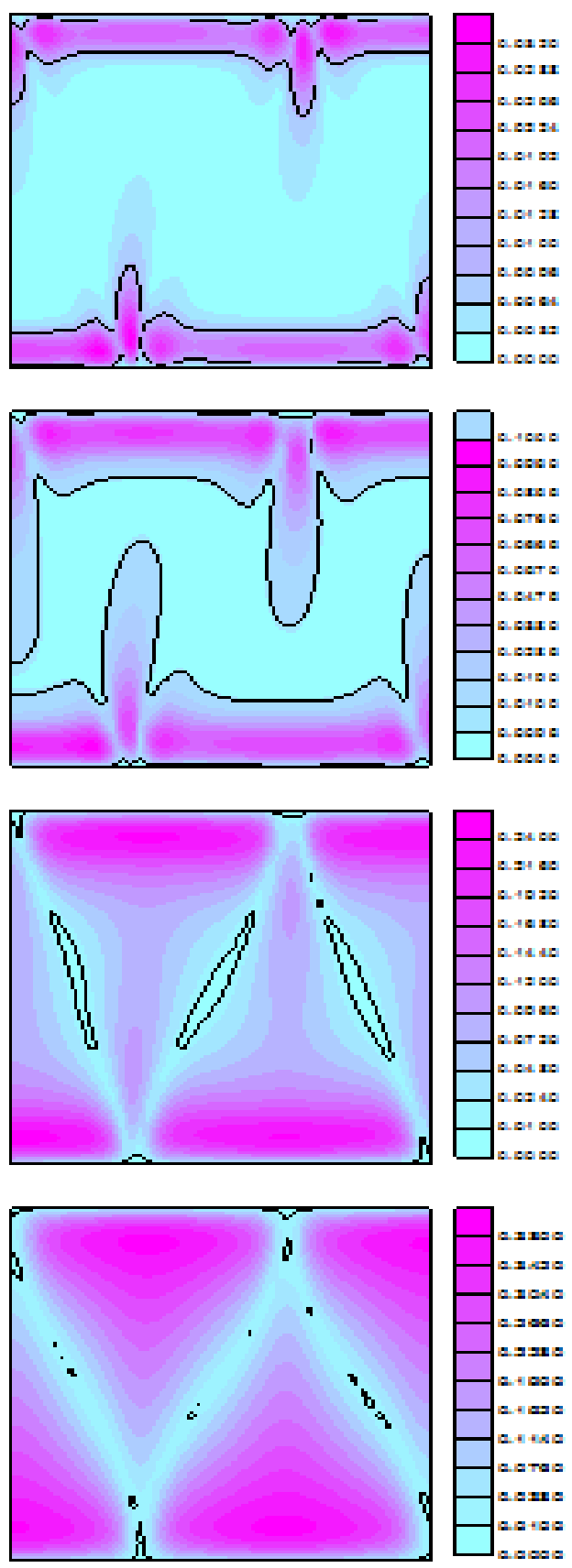

( b )

Fig.(8): The local thermal non-equilibrium (LTNE) at $\mathrm{Ra}^{*}=900$ for the conditions:

(a): $K_{r}=100$ and $\mathrm{H}=100,10,1.0$ and 0.1 from up to down respectively. (b): $\mathrm{H}=100$ and $K_{r}=50,10,1.0$ and0.1 from up to down respectively 


\section{Nusselt Numbers Results}

Fig.(9) demonstrates the effect of modified Rayleigh number on Nusselt numbers for different values of scaled heat transfer coefficient. It is noticed that arising of modified Rayleigh number leads to increase all of Nusselt numbers, also it is noticed that the effect of scaled heat transfer coefficient on total and fluid phase Nusselt numbers was very small due to the high value of thermal conductivity $\operatorname{ratio}\left(K_{r}=100\right)$, in other meaning the high thermal conductivity ratio is caused by high porosity, for this reason, the thermal capacity of the fluid phase is high, then, the thermal content of the fluid phase would has a small response to the change in scaled heat transfer coefficient. The solid phase Nusselt numbers is proportionally increase with scaled heat transfer coefficient because of the reduction in convective resistance between the solid and fluid, hence, more heat exchange between the phases may occur. The similarity between total Nusselt number and fluid phase Nusselt number attributed to the high value of thermal conductivity ratio.
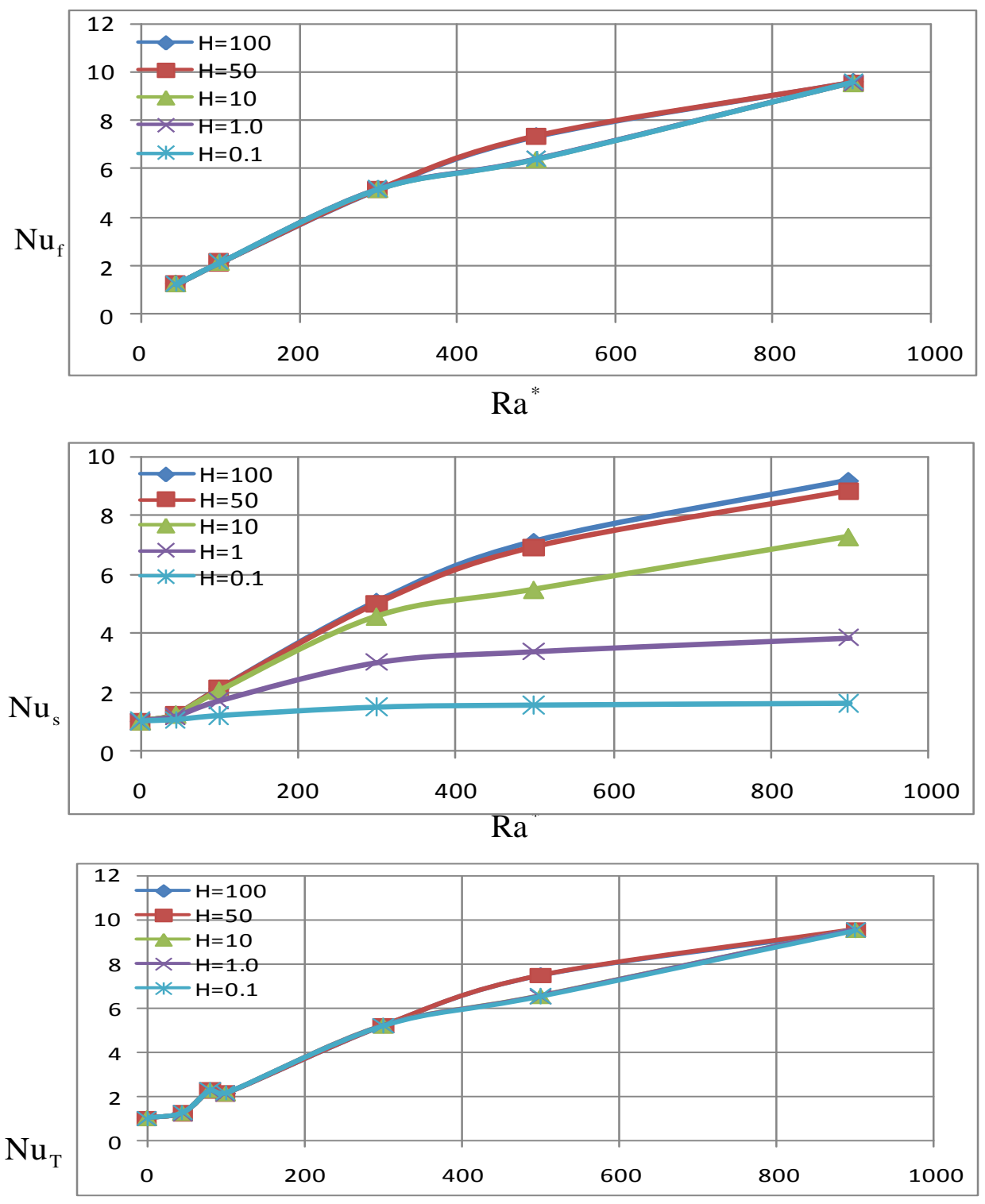

$\mathrm{Ra}^{*}$

Fig(9): The effect of modified Rayleigh number on Nusselt numbers under different values of scaled heat transfer coefficient at $K_{r}=100$. 
Fig.(10) clarifies the effect of modified Rayleigh number on Nusselt numbers for different values of thermal conductivity ratio, all Nusselt numbers increase with increasing of both modified Rayleigh number and thermal conductivity ratio where the increase of thermal conductivity ratio causes more increase in the effective thermal conductivity of the fluid phase and then, more heat transfer through the medium, hence the Nusselt numbers increased also.
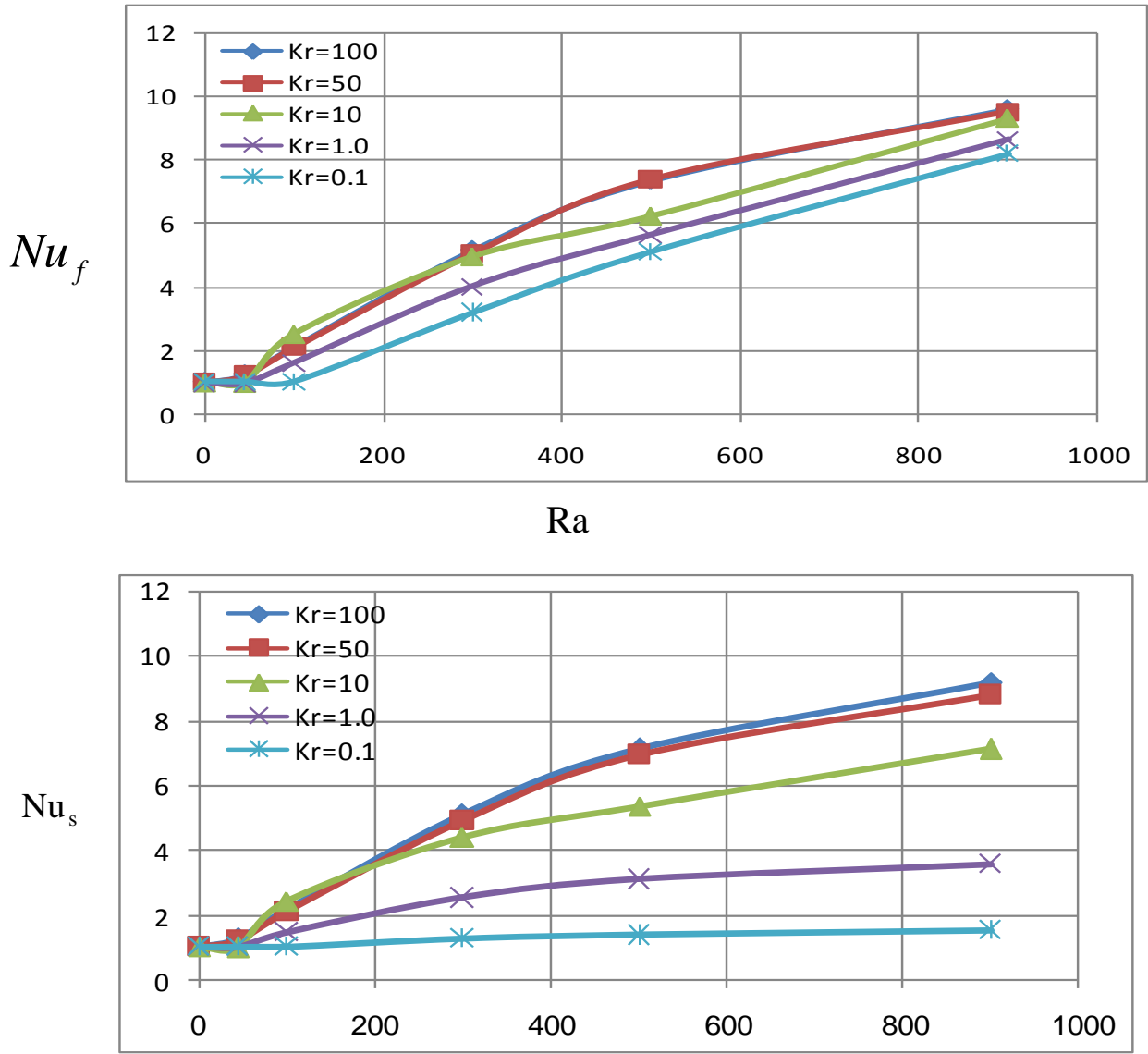

$\mathrm{Ra}^{*}$

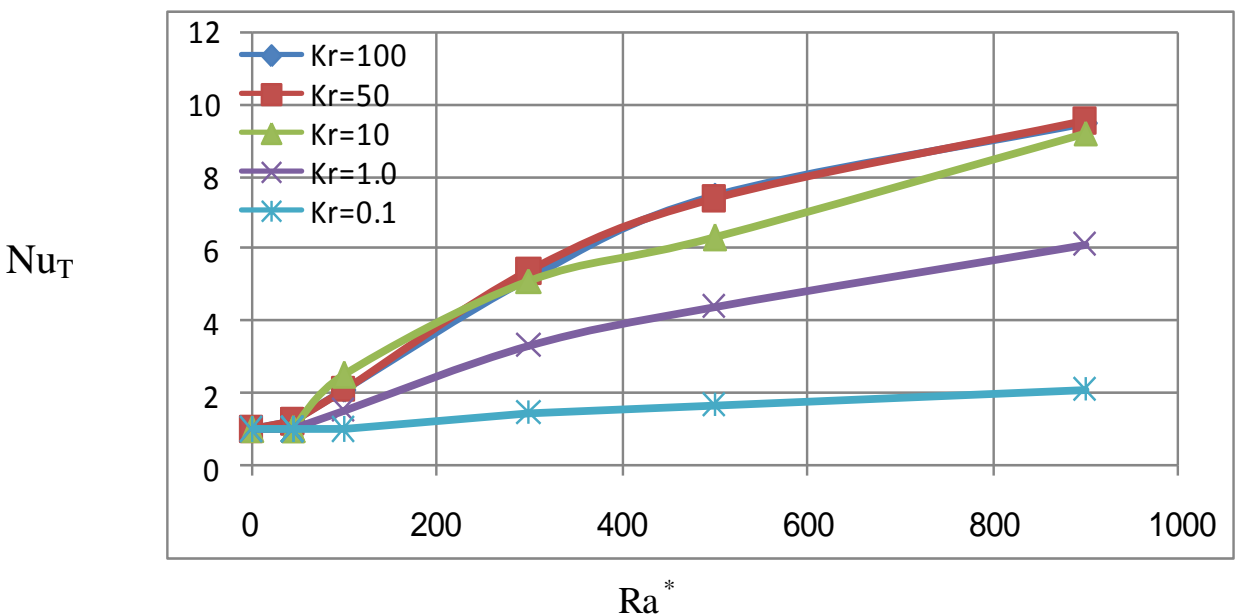

Fig.(10): The effect of modified Rayleigh number on Nusselt numbers under different values of thermal conductivity ratio at $\mathrm{H}=100$. 


\section{Conclusion}

As a conclusion, the effect of thermal conductivity ratio on isograms is more than effect of scaled heat transfer coefficient, the area and strength of non-equilibrium are dependent of scaled heat transfer coefficient more than its depending on thermal conductivity ratio. It is noticed that the high values of thermal conductivity ratio and scaled heat transfer coefficient leads to local thermal equilibrium for the mid values of Rayleigh number, but the local thermal equilibrium becomes invalid for high values of modified Rayleigh number. The increase of modified Rayleigh number causes enlarging of the non-equilibrium spots in addition to increasing the values of LTNE. The effect of scaled heat transfer coefficient is confined to solid phase Nusslet numbers for $K_{r}=100$, while the effect of thermal conductivity ratio comprises all Nusslet numbers (solid, fluid and total).

\section{References}

[1]- Sozen, M. \& Vafai, K., "Analysis of Non-thermal Equilibrium Condensing Flow of a Gas Through a Packed Bed", Int. J. Heat Mass Transfer, Vol. 33, pp1247-1261,(1990).

[2]- Jagjiwanram \& Singh, R., "Effective Thermal Conductivity of Highly Porous Two-phase Systems", Applied Thermal Eng.,Vol.24,pp. 2727-2735, (2004).

[3]- Minkowycs, Haji-Sheikh, W. J., A., Vafai, K., "On Departure from Local Thermal Equilibrium in Porous Media Due to a Rapidly Changing Heat Source: the Sparrow Number", Int. J. Heat Mass Transfer, Vol. 42, pp. 3373-3385, (1999).

[4]- Haddad,O. M., Al-Nimr, M. A., Al-Khateeb, A. N., "Validation of the Local Thermal Equilibrium Assumption in Natural Convection from a Vertical Plate Embedded in Porous Medium: Non-Darcian Model ", Int. J. Heat Mass Transfer, Vol. 47, pp. 20372042, (2004).

[5]- Nnanna, A.C. A., Haji-Sheikh, A., Harris, K. T., "Experimental Study of Local Thermal Non-Equilibrium Phenomena During Phase Change in Porous Media", Int. J. Heat Mass Transfer, Vol. 47, pp. 4365-4375,(2004).

[6]- Baytas, A.C and Pop ,I., "Free convection in a square porous cavity using a thermal nonequilibrium model", Int. J. Thermal science, Vol. 41, pp861-870,(2002).

[7]- Kuriakoz, A. S., "Numerical Study of Natural Convection Heat Transfer in a Porous Cavity with Corrugated Boundaries", Master Thesis, Univ. Mosul, 2006.

[8]- Hwang, J. J. \& Chen, P. Y., "Heat/Mass Transfer in Porous Electrodes of Fuel Cells" , Int. J. Heat Mass Transfer, Vol. 49, pp. 3315-2327, (2006). 